\title{
Designing Peptide-Based HIV Vaccine for Chinese
}

\author{
Jiayi Shu, ${ }^{1,2}$ Xiaojuan Fan, ${ }^{3}$ Jie Ping, ${ }^{3}$ Xia Jin, ${ }^{1,2}$ and Pei Hao ${ }^{3}$ \\ ${ }^{1}$ Viral Disease and Vaccine Translational Research Unit, Institut Pasteur of Shanghai, Chinese Academy of Sciences, \\ Room 507, Building B, Life Science Research Building, 320 Yueyang Road, Shanghai 200031, China \\ ${ }^{2}$ Vaccine Centre, Institut Pasteur of Shanghai, Chinese Academy of Sciences, 320 Yueyang Road, Shanghai 200031, China \\ ${ }^{3}$ Bioinformatics Platform, Institut Pasteur of Shanghai, Chinese Academy of Sciences, Room 405, Building B, \\ Life Science Research Building, 320 Yueyang Road, Shanghai 200031, China \\ Correspondence should be addressed to Xia Jin; xjin@ips.ac.cn and Pei Hao; phao@ips.ac.cn
}

Received 15 April 2014; Accepted 16 June 2014; Published 6 July 2014

Academic Editor: Siyuan Zheng

Copyright (C) 2014 Jiayi Shu et al. This is an open access article distributed under the Creative Commons Attribution License, which permits unrestricted use, distribution, and reproduction in any medium, provided the original work is properly cited.

\begin{abstract}
CD4+ T cells are central to the induction and maintenance of CD8+ T cell and antibody-producing B cell responses, and the latter are essential for the protection against disease in subjects with HIV infection. How to elicit HIV-specific CD4+ T cell responses in a given population using vaccines is one of the major areas of current HIV vaccine research. To design vaccine that targets specifically Chinese, we assembled a database that is comprised of sequences from 821 Chinese HIV isolates and 46 human leukocyte antigen (HLA) DR alleles identified in Chinese population. We then predicted 20 potential HIV epitopes using bioinformatics approaches. The combination of these 20 epitopes has a theoretical coverage of $98.1 \%$ of the population for both the prevalent HIV genotypes and also Chinese HLA-DR types. We suggest that testing this vaccine experimentally will facilitate the development of a CD4+ T cell vaccine especially catered for Chinese.
\end{abstract}

\section{Introduction}

Over 30 million people have died from HIV/AIDS related illnesses since HIV was discovered in the 1980s. There are currently 33 million of HIV carriers [1]. The rate of new infection is still on the rise globally. In China, HIV infection is a great concern, especially in southern part of China, for example, Yunnan, Sichuan, Guangxi, and Xinjiang Provinces, where a large number of infected people are drug users. Additionally, in the regions of Henan, Hubei Provinces where people were infected through illicit blood collection, the rate of infection reached up to $60 \%$ of blood donors [2]. Highly active antiretroviral therapy (HAART), a combination of three or more antiretroviral drugs, is routinely used to treat individuals with HIV infection [3]. It significantly extends the lifespan and improves the quality of life of people infected with HIV but cannot eradicate the virus [4]. The course of treatment is life-long and the medicines are expensive. In developing countries, available antiretroviral drugs are still limited. Therefore, a preventive HIV vaccine is especially needed.
HIV genome is comprised of nine structural (Env, Gag, and $\mathrm{Pol}$ ) and regulatory (Tat, Rev, Nef, Vif, Vpr, and Vpu) genes. The pol gene encodes for reverse transcriptase which is error prone. This leads to high mutation rate, $15-20 \%$ divergence between the nucleic acid sequences of different HIV clades, and $7-12 \%$ variability within each clade [5]. Although the base composition of HIV genome is stable [6], host immune response further increases the HIV nucleotide diversity.

Due to the extreme sequence diversity and high mutation rate of HIV, it has been difficult to develop an efficacious HIV vaccine. A successful HIV vaccine requires inducing neutralizing antibodies and cytotoxic $\mathrm{T}$ cell responses, both of which can only be optimally induced and maintained in the presence of a concurrent $\mathrm{CD} 4+\mathrm{T}$ helper cell response [7]. Despite many years of basic and clinical research, to date, there are only three major human HIV vaccine clinical trials completed. Set up in 1998, AIDSVAX gp120 protein vaccine is the first HIV vaccine going through Phase III trial in human and targeted to induce neutralizing antibody activity. Although antibodies to homologous virus were elicited, they 
failed to neutralize heterologous viruses [8]. In 2004, a Phase IIb trial with Merck's MRKAd5, which is a trivalent vaccine including gag, pol, and nef genes in an adenovirus 5 vector, is designed for inducing cytotoxic $\mathrm{T}$ cell responses $[9,10]$. Despite the induction of significant level of IFN gammaproducing $\mathrm{T}$ cells, the MRKAd5 has increased the risk of HIV acquisition in vaccine recipients and failed to reduce viral load after HIV infection [11]. Later in 2009, a Phase III trial of RV144 HIV-1 vaccine was completed in Thailand, which is a vaccine combination comprised of ALVAC (a vaccine containing genetically engineered versions of gag, env, and pol inserted in canarypox vector) and AIDSVAX (a bivalent gp120 envelope protein vaccine). These vaccines are theoretically capable of eliciting both CD8+ T cell response and neutralizing antibody response. Despite neither vaccine worked alone, in the combination, they unexpectedly lowered the HIV incidence by $31.2 \%$ in vaccine recipients; however, they did not reduce viral load [12]. These large clinical trials have opened new questions and revealed new opportunities for HIV vaccine research, including a rethinking of the need for a vaccine for CD4+ T helper cells.

In order to stimulate a CD4+ T helper cell response, antigens need to be processed and presented through $\mathrm{MHC}$ class II molecules. The form of antigen could be either whole protein or peptide epitopes. A previous study with a subunit vaccine comprised of $18 \mathrm{CD} 4+\mathrm{T}$ helper cell epitopes has demonstrated an efficient induction of robust helper $\mathrm{T}$ cell response in a Phase I clinical trial in Caucasian population [13]. Whether a similar strategy works in Chinese population requires to be tested.

To select antigenic epitopes for a vaccine, one must address several issues. One, HIV exhibits high mutation rates, and thus conserved sequences may be needed to cover a given population. Two, the human leukocyte antigen (HLA) is highly polymorphic, and it restricts the proportion of individuals who will respond to a particular antigen $[14,15]$. To overcome these problems, promising $\mathrm{T}$ cell epitopes that bind to several HLA alleles for maximal population coverage should be selected [16], and a large variety of HIV sequences should be considered in the design of a HIV vaccine.

MHC class II is a heterodimer that is comprised of a monomorphic $\alpha$ and a highly polymorphic $\beta$ chain. There are over 400 class II alleles identified, spreading among HLA-DM, HLA-DO, HLA-DP, HLA-DQ, and HLA-DR loci. Among them DRB1 is the most polymorphic gene, consists of 221 alleles; followed by DPB1 and DQB1 that has 84 and 39 alleles, respectively. Whereas other gene loci may have only 1 or 2 alleles [17]. Therefore, DRB1 is the best choice to optimize MHC II coverage. The frequency of HLA-DRB1 serotype differs among ethnic groups. Within DRB1 allotype, DRB $1 * 11$ and 13 serotypes present in $16 \%$ and $14 \%$ of black population, whereas, in Caucasoid and Chinese, DRB $1 * 07$ and $\mathrm{DRB} 1 * 11$ and $\mathrm{DRB} 1 * 12$ and $\mathrm{DRB} 1 * 15$ appear in the highest percentage [17]. The above evidences support the development of a new HIV vaccine specifically for Chinese population. Such a vaccine should have higher probability in dealing with circulating HIV serotypes in China.

To overcome these complex issues of vaccine design, bioinformatics methods may help to determine common features of vaccine antigens that have potential to deal with divergent population and HIV quasispecies. Specifically, bioinformatics-based approach is the most feasible method in screening a large set of peptide epitopes and selection of promising vaccine antigens. In this study, we extracted $821 \mathrm{HIV}$ sequence and 46 Chinese DRB1 alleles from public information and compiled a database. A combination of 7 public available epitope prediction algorithms was used to screen the database and identify CD4+ T cell epitopes as HIV vaccine antigens. We selected a set of 20 epitopes, which in combination could cover more than $98 \%$ of our target population.

\section{Materials and Methods}

2.1. Data Collection and Methods for Epitope Prediction. In total, $821 \mathrm{HIV}$ whole genome sequences of Chinese population were retrieved from HIV Database (http:// www.hiv.lanl.gov/) [27], and the distribution of 46 HLA-DR alleles (Table 1) was extracted from The Allele Frequency Net Database (AFND) (http://www.allelefrequencies.net/) [28].

Seven existing methods available in Immune Epitope Database (IEDB) [29] for MHC class II binding were used to predict HIV epitopes based on binding affinity between HLA DR types and HIV epitopes. These methods included Consensus method [30], NN-align (netMHCII-2.2) [31], stabilization matrix alignment method (SMM-align) [32], Sturniolo [33], average relative binding (ARB) [34], NetMHCIIpan [35], and Combinatorial library (ComLib) [30].

2.2. Epitope Selection. All epitopes are 15 amino acids in length. To be a potential epitope, it must have a MHC binding affinity threshold of $\mathrm{IC}_{50}=500 \mathrm{nM}$ or below. A selected epitope was removed from the epitope pool before the next prediction. The process is repeated until all epitopes were selected. All calculations of epitope selection process were conducted in INFORSENSE Knowledge Discovery Environment (KDS) software platform [36]. The mathematical model used to calculate the predictive score for each DR allele of known coverage (as listed in Table 1) is the following equations:

$$
\begin{gathered}
S(\alpha)=\sum_{i=1}^{821} \sum_{j=1}^{46} \delta_{i, j}(\alpha) \times C(j), \\
\delta_{i, j}(\alpha)= \begin{cases}1, & \text { if } \alpha \text { in the combination of HIV } \\
\text { sequence } i \text { and DR allele } j \\
0, & \text { Otherwise. }\end{cases}
\end{gathered}
$$

In the first equation (I), $\alpha$ represents the epitope; $C(j)$ is the percentage coverage of number $j \mathrm{DRB} 1$ allele; $\delta(\alpha)$ is the function to indicate whether the epitope exists in the combination of HIV sequence and DR allele, existence scored 1 , and absence scored $0 . S(\alpha)$ is the sum of number of times of the binding of HIV sequence $i$ and DR allele $j$ after being standardized to the proportion of DR allele $j$ in all DRB1 
TABLE 1: The DRB1 allele coverage in Chinese population.

\begin{tabular}{|c|c|c|}
\hline Number & Alleles & Coverage \\
\hline 1 & DRB1 $^{*} 01: 01$ & 0.0145 \\
\hline 2 & $\mathrm{DRB1}^{*}$ 01:02 & 0.0014 \\
\hline 3 & $\mathrm{DRB1}^{*}$ 03:01 & 0.0514 \\
\hline 4 & $\mathrm{DRB1}^{*}$ 03:07 & 0.0009 \\
\hline 5 & DRB1 $^{*}$ 04:01 & 0.0120 \\
\hline 6 & DRB1 $^{*}$ 04:02 & 0.0024 \\
\hline 7 & $\mathrm{DRB}^{*}$ 04:03 & 0.0238 \\
\hline 8 & $\mathrm{DRB1}^{*}$ 04:04 & 0.0082 \\
\hline 9 & $\mathrm{DRB1}^{*}$ 04:05 & 0.0413 \\
\hline 10 & $\mathrm{DRB}^{*}$ 04:06 & 0.0233 \\
\hline 11 & $\mathrm{DRB}^{*}$ 04:07 & 0.0041 \\
\hline 12 & DRB1 ${ }^{*}$ 04:08 & 0.0075 \\
\hline 13 & $\mathrm{DRB1}^{*}$ 04:10 & 0.0030 \\
\hline 14 & $\mathrm{DRB1}^{*}$ 04:17 & 0.0018 \\
\hline 15 & $\mathrm{DRB1}^{*}$ 07:01 & 0.0677 \\
\hline 16 & $\mathrm{DRB1}^{*}$ 08:01 & 0.0018 \\
\hline 17 & $\mathrm{DRB1}^{*}$ 08:02 & 0.0076 \\
\hline 18 & $\mathrm{DRB}^{*}$ 08:03 & 0.0512 \\
\hline 19 & DRB1* 08:04 & 0.0029 \\
\hline 20 & DRB1 $^{*}$ 08:09 & 0.001 \\
\hline 21 & $\mathrm{DRB1}^{*}$ 08:12 & 0.0011 \\
\hline 22 & $\mathrm{DRB1}^{*}$ 09:01 & 0.0490 \\
\hline 23 & $\mathrm{DRB1}^{*} 10: 01$ & 0.0149 \\
\hline 24 & $\mathrm{DRB1}^{*} 11: 01$ & 0.0669 \\
\hline 25 & DRB1*11:03 & 0.0015 \\
\hline 26 & DRB1*11:04 & 0.0154 \\
\hline 27 & DRB1 ${ }^{*} 11: 06$ & 0.0013 \\
\hline 28 & DRB1 ${ }^{*} 12: 01$ & 0.0518 \\
\hline 29 & $\mathrm{DRB1}^{*} 12: 02$ & 0.1048 \\
\hline 30 & DRB1 ${ }^{*} 13: 01$ & 0.0227 \\
\hline 31 & $\mathrm{DRB1}^{*} 13: 02$ & 0.0233 \\
\hline 32 & $\mathrm{DRB1}^{*} 13: 03$ & 0.0029 \\
\hline 33 & DRB1*13:12 & 0.0025 \\
\hline 34 & DRB1 ${ }^{*} 14: 01$ & 0.0214 \\
\hline 35 & $\mathrm{DRB1}^{*} 14: 02$ & 0.0013 \\
\hline 36 & $\mathrm{DRB1}^{*} 14: 03$ & 0.0091 \\
\hline 37 & DRB1 $^{*} 14: 04$ & 0.0078 \\
\hline 38 & DRB1*14:05 & 0.0193 \\
\hline 39 & DRB1 $^{*} 14: 07$ & 0.0023 \\
\hline 40 & DRB1 $^{*} 15: 01$ & 0.1139 \\
\hline 41 & DRB1 $^{*} 15: 02$ & 0.0418 \\
\hline 42 & DRB1 $^{*} 15: 04$ & 0.0013 \\
\hline 43 & $\mathrm{DRB1}^{*} 15: 05$ & 0.0018 \\
\hline 44 & $\mathrm{DRB1}^{*} 16: 01$ & 0.0029 \\
\hline 45 & DRB1*16:02 & 0.0401 \\
\hline 46 & DRB1 $^{*} 16: 05$ & 0.0032 \\
\hline Total & & 0.9520 \\
\hline
\end{tabular}

alleles. All DRB1 alleles included in the study cover $95.2 \%$ all Chinese HLA-DR alleles [28].
We selected epitopes from a combined pool of epitopes through KDS platform using 7 prediction methods from IEDB with a dataset that consisted of 821 circulating HIV genome sequences in China and 46 Chinese HLA-DRB1 alleles. The epitopes bind to MHC class I molecules that were removed first, and then the value of $\mathrm{IC}_{50}$ was considered. Next, we ranked all epitopes based on the coverage score (the higher the better coverage in HIV genome and DRHLA alleles). After an epitope has been selected, it was removed from the database before next selection. This process was repeated until 20 epitopes were selected. The workflow diagram of this procedure was illustrated in Figure 1.

\section{Results}

3.1. The Coverage Distribution of HLA-DR of Chinese Population. A total of 46 HLA-DR alleles were identified from AFND (Table 1). The alleles were listed and its coverage in Chinese population was given. The table showed the coverage ranged from $0.1 \%(\mathrm{DRB} 1 * 08: 09)$ to $6.77 \%(\mathrm{DRB} 1 * 07: 01)$ and in a total of $95.2 \%$ of the Chinese population. The sample population comprises 1704 individuals of the Han ethnicity. This information was obtained from ten regions within the mainland, China, and two other regions, Hong Kong and Singapore, where Chinese ethnicity dominates. Among them, the DRB1-02, -05, and -06 genotypes were not detected.

3.2. The Diversity of Epitope Coverage. With a combination of 7 existing epitope prediction methods in IEDB, using database comprised of 46 different DRB1 alleles and 821 full genome sequences of HIV isolates circulating in China, we then predicted $38,460,402$ potential epitopes. After duplicates were removed, 21,007,527 potential epitopes remained. We scored these epitopes based on the allele coverage and total coverage score, which was in general normally distributed. As shown in Figure 2, most epitopes displayed low coverage scores, 0.1 or lower; the highest epitope count reached approximately 3000 .

3.3. HIV Epitopes Specifically for Chinese Population. By using the methods described above, we obtained 20 epitopes, which in theory covered all 46 DRB1 allelic genotypes and 821 Chinese HIV sequences (Table 2). All 20 epitopes were selected for binding to MHC class II and absence of binding to MHC class I. Table 2 listed the amino acid sequences of the 20 epitopes, their location in HIV-1 gene, their percentage of coverage in $\mathrm{HIV}-1$ genome sequences from $4 \%$ to $43 \%$, the proportion in the HLA-DR allele sequences between $52 \%$ and $100 \%$, and the total coverage in both sequences as low as $4 \%$ and the highest at $41 \%$. One single epitope WIILGLNKIVRMYSP covered $41 \%$ of both DRB1 and Chinese specific HIV-1 genome sequences, which is of note. This epitope had been reported before [18]. In fact, 4 other predicted epitopes (LNKIVRMYSPTSILD, GFPVRPQVPLRPMTY, VDRFYKTLRAEQASQ, and LYKYKVVKIEPLGVA) have also been published previously $[22-24,26]$ and 4 peptide sequences (PVVSTQLLLNGSLAE, 
(1)

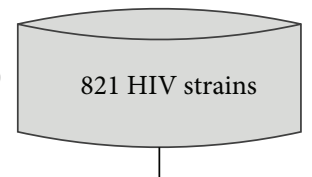

(2)

(3)

(4)

(5)

(6)

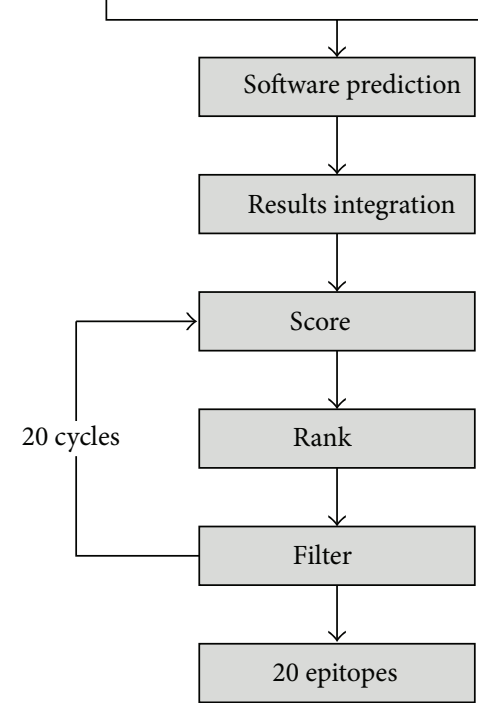

FIGURE 1: A flowchart illustrates procedures for CD4+T cell epitope prediction. (1) Using KDS platform with datasets of 821 circulating HIV-1 strains and 46 HLA-DRB1 alleles in Chinese population; (2) the software predicted possible epitopes by 7 known methods from the IEDB database; (3) all results were combined and scored using (I) and (II); (4) the epitopes were ranked according to the score; (5) the epitope with the top score and the lowest $\mathrm{IC}_{50}$ value was selected; (6) the selected epitope was then removed from the epitope pool; (7) steps 4-6 were repeated until all 20 epitopes fulfilled the criteria that were selected.

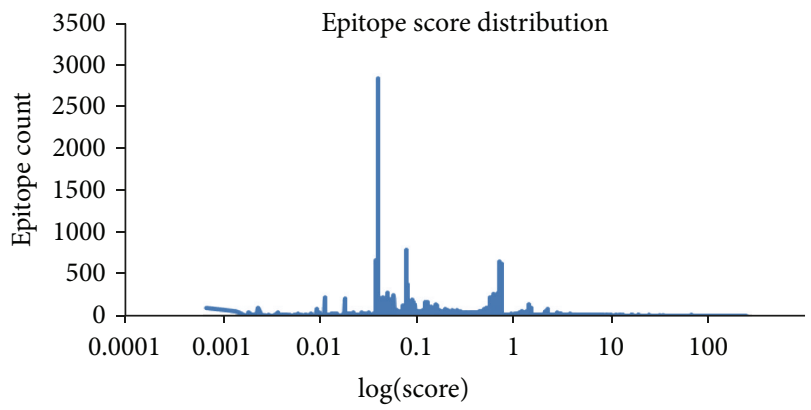

FIGURE 2: The distribution of epitope coverage score. The epitope coverage scores (log-transformed) were plotted on the horizontal axis against the frequency of epitope count on the vertical axis. Most of the log score localized to the region between 0.01 and 1 .

LRIIFAVLSIVNRVR, ILDLWVYHTQGYFPD, and YKRWIILGLNKIVRM) were reported in patents before [19-21, 25], whereas the remaining 11 epitopes have never been reported. All 20 epitopes together provided 98.1\% coverage in HIV genome and HLA-DR alleles. These predicted epitopes were found in HIV-1 gag, env, pol, and nef genes. Six of them were in gag gene, 6 in env, 2 in pol, and 6 in nef.

We then applied the new method to a previously published HIV vaccine comprised of T helper epitopes and tested in clinical trial [13]. The table listed 17 epitopes, from gag, pol, env, and vpu genes. One published epitope that has a HIA binding $\mathrm{IC}_{50}$ above our threshold of 500, Env 566 IKQFINMWQEVKAMY, was not listed. For these epitopes, HIV coverage is from $2 \%$ to $43 \%$, DR coverage is between $35 \%$ and $98 \%$, and specific coverage is at highest of $41 \%$ and in sum of $69 \%$.

\section{Discussion}

In this paper, we described a novel method for designing a peptide-based $\mathrm{T}$ helper cell vaccine for HIV, which is specific for Chinese HIV strains and Chinese MHC class II genotypes. The current method has several advantages. First, our methodology of epitope prediction is easily accessible to public use. In fact, it is a combination of all seven existing methods publically available in IEDB. The IEDB database comprises a series of most up-to-date and evidence based methods specifically created for the prediction of MHC restricted T cell epitopes. In contrast to other studies that only used one of the methods, we used them all for more accurate prediction of MHC class II restricted T helper epitopes.

So far, there are three major types of bioinformatics methods for the prediction of MHC class II restricted T helper cell epitopes. One is called matrix alignment algorithm, and these are SMM, ARB, and Sturniolo methods. This algorithm uses published $\mathrm{T}$ cell epitopes and their respective binding affinity to MHC class II, in terms of the $\mathrm{IC}_{50}$ value, to determine epitopes. The other relies on machine learning, and NNalign and NetMHCIIpan methods belong to this category. New sequences are subjected to computer simulated models to predict whether any epitopes can bind to a particular MHC II to high enough affinity. The third type combines several methods together to predict epitopes. These include Consensus method and ComLib method.

Consensus method was reported to provide highest true positive rate, followed by $\mathrm{NN}$-align and ARB [37]. NetMHCIIpan performed the best among all other panspecific methods for MHC class II with varied experimental settings [38]. NN-align performs especially well in handling large dataset among all other machine learning methods and in combination with ARB outperforms the use of $\mathrm{NN}$-align alone [30].

In this study, we used all above seven methods simultaneously, scored the potential epitopes independently, and then used $\mathrm{IC}_{50}$ value as a filter to select $\mathrm{T}$ cell epitopes that have the broadest population coverage. Our method did not use all 8 IEDB recommended methods but integrated 7 of the IEDB methods because the 8th IEDB method is an integration of the other seven and thus not an independent measurement. The method we used could be considered as "greedy" algorithm in the bioinformatics field, which predicts the best epitope among all in a pool of potential epitopes. Thus, we believe an integrated method that uses a combination of all seven original algorithms might be the best to predict more accurately MHC class II epitopes.

Another unique feature of our study is that we designed candidate helper $\mathrm{T}$ cell vaccine targets specifically to the 
TABLE 2: Predicted HIV T helper cell epitopes for Chinese population.

\begin{tabular}{|c|c|c|c|c|c|c|}
\hline Amino acid sequences & $\begin{array}{c}\text { Protein } \\
\text { destination }\end{array}$ & $\mathrm{HIV}^{2}{ }^{2}$ & $\mathrm{DR} \%^{3}$ & $\begin{array}{c}\text { Total } \\
\text { coverage }^{4}\end{array}$ & $\begin{array}{c}\text { Specific } \\
\text { coverage }^{5}\end{array}$ & Reference $^{6}$ \\
\hline WIILGLNKIVRMYSP & Gag 265 & $43 \%$ & $89 \%$ & $41 \%$ & $40.72 \%$ & Younes et al., 2003 [18] \\
\hline PVVSTQLLLNGSLAE & Env 262 & $38 \%$ & $74 \%$ & $34 \%$ & $27.73 \%$ & August et al., 2013 [19] \\
\hline VQMAVFIHNFKRKGG & Pol 892 & $24 \%$ & $93 \%$ & $23 \%$ & $9.79 \%$ & NA (IEDB) \\
\hline LRIIFAVLSIVNRVR & Env 702 & $24 \%$ & $96 \%$ & $26 \%$ & $4.39 \%$ & Sette et al., 2005 [20] \\
\hline ILDLWVYHTQGYFPD & Nef 127 & $12 \%$ & $63 \%$ & $10 \%$ & $5.37 \%$ & Sette et al., 2002 [21] \\
\hline LNKIVRMYSPTSILD & Gag 284 & $25 \%$ & $100 \%$ & $25 \%$ & $1.81 \%$ & Korber et al., 2001 [22] \\
\hline WGIKQLQARVLAVER & Env 588 & $22 \%$ & $87 \%$ & $20 \%$ & $1.25 \%$ & NA \\
\hline GAFDLSFFLKEKGGL & Nef 91 & $4 \%$ & $63 \%$ & $4 \%$ & $1.20 \%$ & NA \\
\hline VDRFYKTLRAEQATQ & Gag 297T & $15 \%$ & $98 \%$ & $15 \%$ & $1.17 \%$ & NA \\
\hline GFPVRPQVPLRPMTY & Nef 85 & $9 \%$ & $65 \%$ & $6 \%$ & $0.84 \%$ & Korber et al., 2002 [23] \\
\hline TPGIRYQYNVLPQGW & Pol 295 & $23 \%$ & $93 \%$ & $22 \%$ & $0.78 \%$ & NA \\
\hline VDRFYKTLRAEQASQ & Gag 297S & $15 \%$ & $98 \%$ & $15 \%$ & $0.71 \%$ & Bozzacco et al., 2012 [24] \\
\hline RQLLSGIVQQQSNLL & Env 549 & $27 \%$ & $83 \%$ & $26 \%$ & $0.56 \%$ & NA \\
\hline GLIYSKKRQEILDLW & Nef 117 & $6 \%$ & $67 \%$ & $5 \%$ & $0.50 \%$ & NA \\
\hline KPCVKLTPLCVTLNC & Env 126 & $17 \%$ & $89 \%$ & $16 \%$ & $0.28 \%$ & NA \\
\hline YKRWIILGLNKIVRM & Gag 272 & $43 \%$ & $89 \%$ & $41 \%$ & $0.24 \%$ & Sette et al., 2002 [25] \\
\hline PLTFGWCFKLVPVDP & Nef 144 & $11 \%$ & $52 \%$ & $11 \%$ & $0.21 \%$ & NA \\
\hline FGWCFKLVPVDPREV & Nef 147 & $4 \%$ & $93 \%$ & $4 \%$ & $0.24 \%$ & NA \\
\hline CKQIIKQLQPALQTG & Gag 67 & $8 \%$ & $98 \%$ & $8 \%$ & $0.16 \%$ & NA \\
\hline LYKYKVVKIEPLGVA & Env 489 & $6 \%$ & $100 \%$ & $6 \%$ & $0.14 \%$ & Dzuris et al., 2001 [26] \\
\hline Total specific coverage ${ }^{7}$ & & & & & $98.1 \%$ & \\
\hline
\end{tabular}

${ }^{1}$ The location of epitopes on HIV viral gene products and the first amino acid of the viral gene product.

${ }^{2}$ The epitope sequence presented in the proportion of 821 HIV genome sequences.

${ }^{3}$ The epitope sequence presented in the proportion of $46 \mathrm{DR}$ alleles.

${ }^{4}$ The ratio of the epitope appeared in both 821 HIV genome and DR allele sequences.

${ }^{5}$ Calculated based on the coverage of the epitope in the rest of the dataset after removing the preceding epitope.

${ }^{6}$ Reference where the epitope had been published. NA: not available in published literature.

${ }^{7}$ Sum of specific coverage for all 20 epitopes.

Chinese population. Most common world circulating HIV subtypes are $\mathrm{B}$ and $\mathrm{C}$, and recombinant forms are $\mathrm{AE}$ and AG. In contrast, the common subtypes are $B$ and recombinant forms are $\mathrm{BC}$ and $\mathrm{AE}$ in China [27, 39, 40]. We extracted all 821 subtypes of HIV-1 strains which are mostly subtypes B and $\mathrm{C}$ for developing a highly specific vaccine for Chinese population. As $\mathrm{T}$ helper cell epitopes are recognized through MHC class II, and that Chinese exhibit divergence DRB1 alleles, we also included 46 published Chinese HLA-DRB1 genotypes into our prediction.

In comparison to a previous paper that selected MHC class II binders according to the binding affinity to multiple HLA-DR subtypes [13], we focused on DRB1 alleles which are most polymorphic among human MHC class II loci and thus directed our study to be more specific and increased possibility to induce $\mathrm{T}$ cell responses specifically for Chinese.

One limitation in our study, as shown in Table 1, is that DRB1 genotypes 2, 5, and 6 were not included. This is due to a lack of publication of any information on $\mathrm{DRB} 1 * 02,05$, and 06 . Therefore, our dataset represents what is currently available; that is, there are only 46 DRB1 alleles in Chinese population.

By using our method, we obtained 20 helper $\mathrm{T}$ cell epitopes which covered $98.1 \%$ of HIV strains known to have been circulating in China and all Chinese HLA-DR genotypes. There are limited studies that have tested designed peptide $\mathrm{T}$ helper vaccine in humans. In a published paper that contains $18 \mathrm{~T}$ helper epitopes [13], our combination of epitope predication methods found that these epitopes covered $69 \%$ of Chinese HIV genomes (Table 3). In a different population that is predominantly Caucasian, these epitopes combined have a $100 \%$ coverage. Thus, the difference in the coverage may suggest our predicting method is more specific for Chinese population, and our epitopes are better potential HIV vaccine candidate for Chinese. Furthermore, 9 epitopes we obtained have been published before and 11 are not. Thus, we both have the empirical evidence to support that our allelic specific peptides have the potential to stimulate $\mathrm{T}$ cell responses and new epitopes to suggest that our prediction is innovative. 
TABLE 3: Using novel algorism to calculate the coverage of epitopes in a published T helper vaccine for Chinese population.

\begin{tabular}{|c|c|c|c|c|}
\hline Amino acid sequence & Protein destination $^{1}$ & HIV $^{2}$ & $\mathrm{DR} \%^{3}$ & Specific coverage ${ }^{4}$ \\
\hline FRKYTAFTIPSINNE & Pol 303 & $14 \%$ & $98 \%$ & $13 \%$ \\
\hline EKVYLAWVPAHKGIG & Pol 711 & $3 \%$ & $98 \%$ & $3 \%$ \\
\hline GEIYKRWIILGLNKI & Gag 294 & $20 \%$ & $87 \%$ & $18 \%$ \\
\hline KRWIILGLNKIVRMY & Gag 298 & $43 \%$ & $89 \%$ & $41 \%$ \\
\hline GAVVIQDNSDIKVVP & Pol 989 & $21 \%$ & $57 \%$ & $12 \%$ \\
\hline YRKILRQRKIDRLID & Vpu 31 & $2 \%$ & $89 \%$ & $2 \%$ \\
\hline QKQITKIQNFRVYYR & Pol 956 & $19 \%$ & $98 \%$ & $19 \%$ \\
\hline SPAIFQSSMTKILEP & Pol 335 & $11 \%$ & $93 \%$ & $11 \%$ \\
\hline QHLLQLTVWGIKQLQ & Env 729 & $23 \%$ & $83 \%$ & $21 \%$ \\
\hline AETFYVDGAANRETK & Pol 619 & $7 \%$ & $41 \%$ & $2 \%$ \\
\hline QGQMVHQAISPRTLN & Gag 171 & $3 \%$ & $85 \%$ & $3 \%$ \\
\hline WAGIKQEFGIPYNPQ & Pol 874 & $3 \%$ & $35 \%$ & $1 \%$ \\
\hline KVYLAWVPAHKGIGG & Pol 712 & $3 \%$ & $93 \%$ & $2 \%$ \\
\hline KTAVQMAVFIHNFKR & Pol 915 & $24 \%$ & $83 \%$ & $22 \%$ \\
\hline EVNIVTDSQYALGII & Pol 674 & $24 \%$ & $57 \%$ & $16 \%$ \\
\hline WEFVNTPPLVKLWYQ & Pol 596 & $22 \%$ & $91 \%$ & $22 \%$ \\
\hline HSNWRAMASDFNLPP & Pol 758 & $11 \%$ & $57 \%$ & $7 \%$ \\
\hline Total specific coverage $e^{5}$ & & & & $69 \%$ \\
\hline
\end{tabular}

${ }^{1}$ The epitopes were selected from a published paper.

Data in columns 2-5 were calculated using the same method as in Table 2.

There was one core epitope WIILGLNKIVRMY, appeared in both studies, showing very high HIV, HLA-DR, and specific coverage. The Gag epitope with two amino acids modification WIILGLNKIVRMYSP was reported to stimulate strong $\overline{\mathrm{CD} 4+\mathrm{T} \text { responses }}$ [27]; another variant of the same epitope KRWIILGLNKIVRMY exhibited superior HLA-DR binding capacity [13]. Another difference between our study and that published is that our epitopes consisted of those in nef gene but not $v p u$ gene, whereas Walker's study did not cover nef but $v p u$. These comparisons suggest that a vaccine designed predominantly for Caucasian may not be optimal for Chinese population. One epitope, for instance, Env 566 (IKQFINMWQEVKAMY) [13], given in Walker's paper, was not picked up in our study.

Our method predicted epitopes, in theory, together covered $98.1 \%$ of HIV-1 genome and Chinese specific DRB1 alleles. In comparison, Walker's study reported $18 \mathrm{~T}$ helper cell epitopes that cover $100 \%$ of the global population. By using a prediction algorithm which based mostly on HLA supertypes [13]. However, when submitted to our new prediction method, the same epitopes only achieved 69\% of coverage of the Chinese population. The discrepancy in methods for prediction may give different results. Further experimental evidence is required to find out whether our method is more accurate.

The allele coverage of DRB1 for Chinese was based on 1704 subjects of whom 1569 were from mainland China and 135 were from Hong Kong and Singapore. All Chinese allele data regarding DRB1 frequencies were extracted from AFND, and all 1704 subjects were Chinese Han ethnics. There is no information on other minor national groups in China available. This may lead to inaccuracy in prediction of helper $\mathrm{T}$ cell epitopes for the Chinese. Larger sample size may improve the quality of our prediction.

\section{Conclusions}

In this study, we report a novel bioinformatics method for designing peptide epitope based T helper vaccine for HIV. We suggest further in vitro and in vivo experiments to be performed to test the immunogenicity of this vaccine and improvement of method of prediction to be made when necessary.

\section{Conflict of Interests}

The authors declare that there is no conflict of interests regarding the publication of this paper.

\section{Authors' Contribution}

Jiayi Shu and Xiaojuan Fan contributed equally to this work.

\section{Acknowledgment}

This work is supported by the Grant of National Major Scientific and Technological Special Project for "Significant New Drugs Development" during the Twelfth Five-Year Plan Period (2013ZX10001002002002). 


\section{References}

[1] Joint, "Global report fact sheet: The global AIDS epidemic," Edited by HIV/AIDS UNPo, 2010, http://www.unaids.org/documents/20101123_FS_Global_em_en.pdf.

[2] Y. X. Yan, Y. Q. Gao, X. Sun et al., "Prevalence of hepatitis C virus and hepatitis B virus infections in HIV-positive Chinese patients," Epidemiology and Infection, vol. 139, no. 3, pp. 354360, 2011.

[3] D. D. Ho, A. U. Neumann, A. S. Perelson, W. Chen, J. M. Leonard, and M. Markowitz, "Rapid turnover of plasma virions and CD4 lymphocytes in HIV-1 infection," Nature, vol. 373, no. 6510, pp. 123-126, 1995.

[4] F. J. Palella Jr., K. M. Delaney, A. C. Moorman et al., "Declining morbidity and mortality among patients with advanced human immunodeficiency virus infection," The New England Journal of Medicine, vol. 338, no. 13, pp. 853-860, 1998.

[5] A. S. de Groot, B. Jesdale, W. Martin et al., "Mapping crossclade HIV-1 vaccine epitopes using a bioinformatics approach," Vaccine, vol. 21, pp. 27-30, 2003.

[6] A. C. van der Kuyl and B. Berkhout, "The biased nucleotide composition of the HIV genome: a constant factor in a highly variable virus," Retrovirology, vol. 9, article 92, 2012.

[7] B. D. Walker and D. R. Burton, "Toward an AIDS vaccine," Science, vol. 320, no. 5877, pp. 760-764, 2008.

[8] N. M. Flynn, D. N. Forthal, C. D. Harro et al., "Placebocontrolled phase 3 trial of a recombinant glycoprotein 120 vaccine to prevent HIV-1 infection," Journal of Infectious Diseases, vol. 191, no. 5, pp. 654-665, 2005.

[9] S. P. Buchbinder, D. V. Mehrotra, A. Duerr et al., "Efficacy assessment of a cell-mediated immunity HIV-1 vaccine (the Step Study): a double-blind, randomised, placebo-controlled, test-of-concept trial," The Lancet, vol. 372, no. 9653, pp. 18811893, 2008.

[10] M. J. McElrath, S. C. de Rosa, Z. Moodie et al., "HIV-1 vaccineinduced immunity in the test-of-concept Step Study: a casecohort analysis," The Lancet, vol. 372, no. 9653, pp. 1894-1905, 2008.

[11] A. S. Fauci, M. I. Johnston, C. W. Dieffenbach et al., "HIV vaccine research: the way forward," Science, vol. 321, no. 5888, pp. 530-532, 2008.

[12] S. Rerks-Ngarm, P. Pitisuttithum, S. Nitayaphan et al., "Vaccination with ALVAC and AIDSVAX to prevent HIV-1 infection in Thailand," The New England Journal of Medicine, vol. 361, no. 23, pp. 2209-2220, 2009.

[13] L. E. Walker, L. Vang, X. Shen et al., "Design and preclinical development of a recombinant protein and DNA plasmid mixed format vaccine to deliver HIV-derived T-lymphocyte epitopes," Vaccine, vol. 27, no. 50, pp. 7087-7095, 2009.

[14] V. Brusic and J. T. August, "The changing field of vaccine development in the genomics era," Pharmacogenomics, vol. 5, no. 6, pp. 597-600, 2004.

[15] I. G. Ovsyannikova, R. M. Jacobson, and G. A. Poland, "Variation in vaccine response in normal populations," Pharmacogenomics, vol. 5, no. 4, pp. 417-427, 2004.

[16] N. C. Toussaint and O. Kohlbacher, "OptiTope-a web server for the selection of an optimal set of peptides for epitope-based vaccines," Nucleic Acids Research, vol. 37, no. 2, pp. W617-W622, 2009.

[17] S. G. E. Marsh, P. Parham, and L. D. Barber, "No. Part 11. HLADR," in The HLA Facts Book, vol. 1st, pp. 330-390, Academic Press, San Diego, Calif, USA, 2000.
[18] S. Younes, B. Yassine-Diab, A. R. Dumont et al., "HIV-1 viremia prevents the establishment of interleukin 2-producing HIVspecific memory $\mathrm{CD}^{+} \mathrm{T}$ cells endowed with proliferative capacity," The Journal of Experimental Medicine, vol. 198, no. 12, pp. 1909-1922, 2003.

[19] J. T. August, G. G. Simon, T. W. Tan, A. M. Khan, and Y. Hu, "Human immunodeficientcy virus (HIV-1) highly conserved and low variant sequences as targets for vaccine and diagnostic applications," United States Patent Application US2013/0195904 A1, National University of Singapore, The Johns Hopkins University, edited by Office USP, 2013.

[20] A. Sette, J. Sidney, S. Southwood et al., Inducint Cellular Immune Responses to Human Immunodeficiency Virus-1 Using Peptide and Uncleic Acid Compositions, vol. 20050271676, Epimmune Inc, San Diego, Calif, USA, 2005, Edited by Office USP.

[21] A. Sette, J. Sidney, and S. Southwood, HLA Class I and II Binding Peptides and Their Uses, vol. WO2003040165A2, Epimmune Inc., New York, NY, USA, 2002.

[22] B. T. M. Korber, R. Koup, B. D. Walker et al., HIV Molecular Immunology, vol. LA-UR 02, Theoretical Biology and Biophysics, Los Alamos, NM, USA, 2001, edited by J. A. Bradac.

[23] B. T. M. Korber, R. Koup, B. D. Walker et al., "HIV molecular immunology," in Theoretical Biology and Biophysics, J. A. Bradac, Ed., 2002.

[24] L. Bozzacco, H. Q. Yu, J. Dengjel et al., "Strategy for identifying dendritic cell-processed CD4+ T cell epitopes from the HIV Gag p24 protein," PLoS ONE, vol. 7, no. 7, Article ID e41897, 2012.

[25] A. Sette, J. Sidney, and S. Southwood, "Identification of broadly reactive DR restricted epitopes," Tech. Rep. WO 1999061916 Al, Epimmune, San Diego, Calif, USA, 2002.

[26] J. L. Dzuris, J. Sidney, H. Horton et al., "Molecular determinants of peptide binding to two common rhesus macaque major histocompatibility complex class II molecules," Journal of Virology, vol. 75, no. 22, pp. 10958-10968, 2001.

[27] C. Kuiken, B. Korber, and R. W. Shafer, "HIV sequence databases," AIDS Reviews, vol. 5, no. 1, pp. 52-61, 2003.

[28] F. F. Gonzalez-Galarza, S. Christmas, D. Middleton, and A. R. Jones, "Allele frequency net: A database and online repository for immune gene frequencies in worldwide populations," Nucleic Acids Research, vol. 39, no. 1, pp. D913-D919, 2011.

[29] R. Vita, L. Zarebski, J. A. Greenbaum et al., "The immune epitope database 2.0," Nucleic Acids Research, vol. 38, pp. D854D862, 2010.

[30] P. Wang, J. Sidney, Y. Kim et al., "Peptide binding predictions for HLA DR, DP and DQ molecules," BMC Bioinformatics, vol. 11, article 568, 2010.

[31] M. Nielsen and O. Lund, "NN-align. An artificial neural network-based alignment algorithm for MHC class II peptide binding prediction," BMC Bioinformatics, vol. 10, article 296, 2009.

[32] M. Nielsen, C. Lundegaard, and O. Lund, "Prediction of MHC class II binding affinity using SMM-align, a novel stabilization matrix alignment method," BMC Bioinformatics, vol. 8, article 238, 2007.

[33] T. Sturniolo, E. Bono, J. Ding et al., "Generation of tissuespecific and promiscuous HLA ligand databases using DNA microarrays and virtual HLA class II matrices," Nature Biotechnology, vol. 17, no. 6, pp. 555-561, 1999.

[34] H. H. Bui, J. Sidney, B. Peters et al., "Automated generation and evaluation of specific MHC binding predictive tools: ARB matrix applications," Immunogenetics, vol. 57, no. 5, pp. 304-314, 2005. 
[35] M. Nielsen, C. Lundegaard, T. Blicher et al., "Quantitative predictions of peptide binding to any HLA-DR molecule of known sequence: NetMHCIIpan," PLoS Computational Biology, vol. 4, no. 7, Article ID e1000107, 2008.

[36] Q. Lu, P. Hao, V. Curcin et al., "KDE bioscience: platform for bioinformatics analysis workflows," Journal of Biomedical Informatics, vol. 39, no. 4, pp. 440-450, 2006.

[37] P. Wang, J. Sidney, C. Dow, B. Mothé, A. Sette, and B. Peters, "A systematic assessment of MHC class II peptide binding predictions and evaluation of a consensus approach," PLoS Computational Biology, vol. 4, no. 4, Article ID e1000048, 2008.

[38] L. M. Zhang, K. Udaka, H. Mamitsuka, and S. F. Zhu, "Toward more accurate pan-specific MHC-peptide binding prediction: a review of current methods and tools," Briefings in Bioinformatics, vol. 13, no. 3, pp. 350-364, 2011.

[39] X. L. Yu, L. Yuan, Y. Huang et al., "Susceptibility of HIV-1 subtypes B', CRF07_BC and CRF01_AE that are predominantly circulating in China to HIV-1 entry inhibitors," PLoS ONE, vol. 6, no. 3, Article ID e17605, 2011.

[40] W. Wang, S. Jiang, S. Li et al., "Identification of subtype B, multiple circulating recombinant forms and unique recombinants of HIV type 1 in an MSM cohort in China," AIDS Research and Human Retroviruses, vol. 24, no. 10, pp. 1245-1254, 2008. 

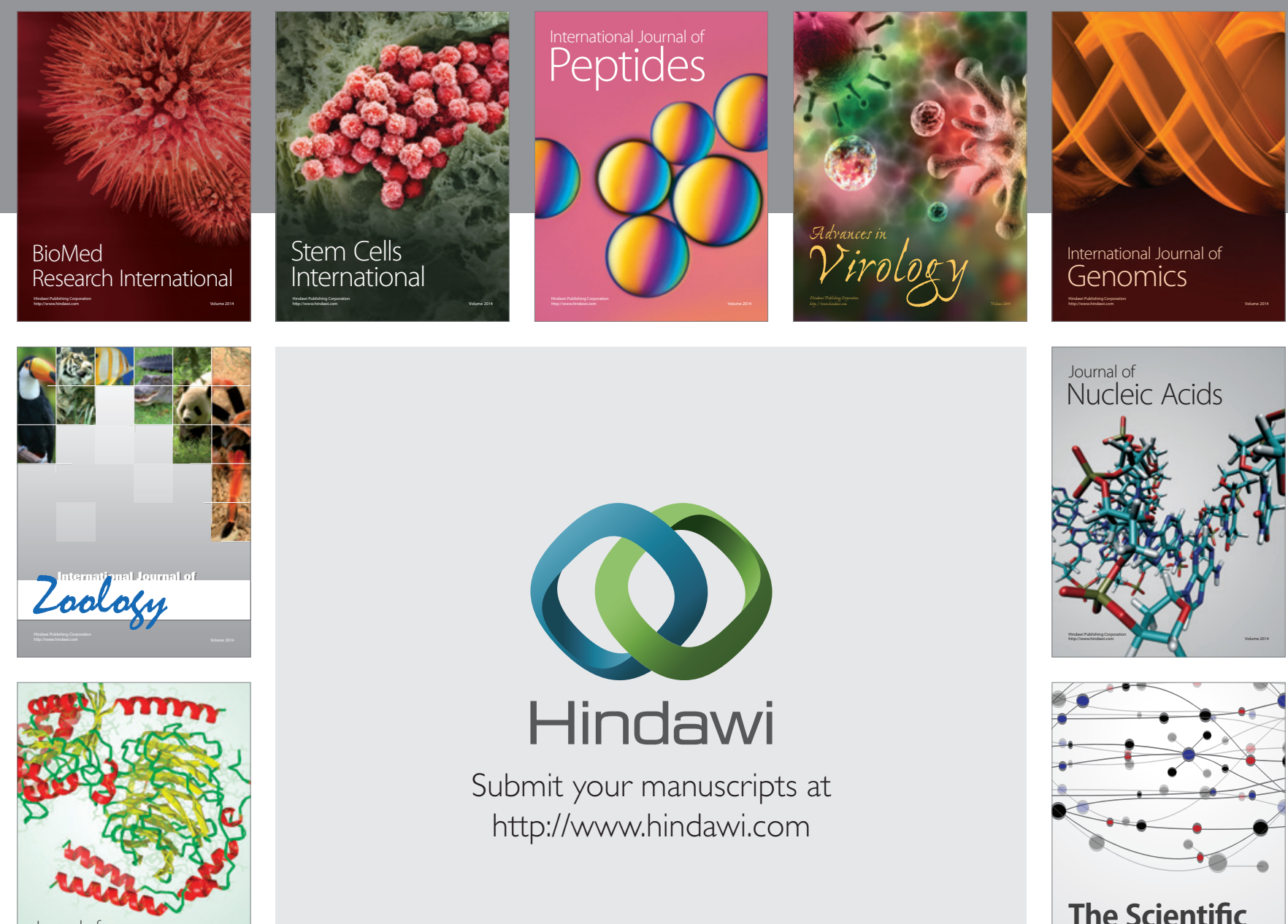

Submit your manuscripts at

http://www.hindawi.com

Journal of
Signal Transduction


The Scientific World Journal
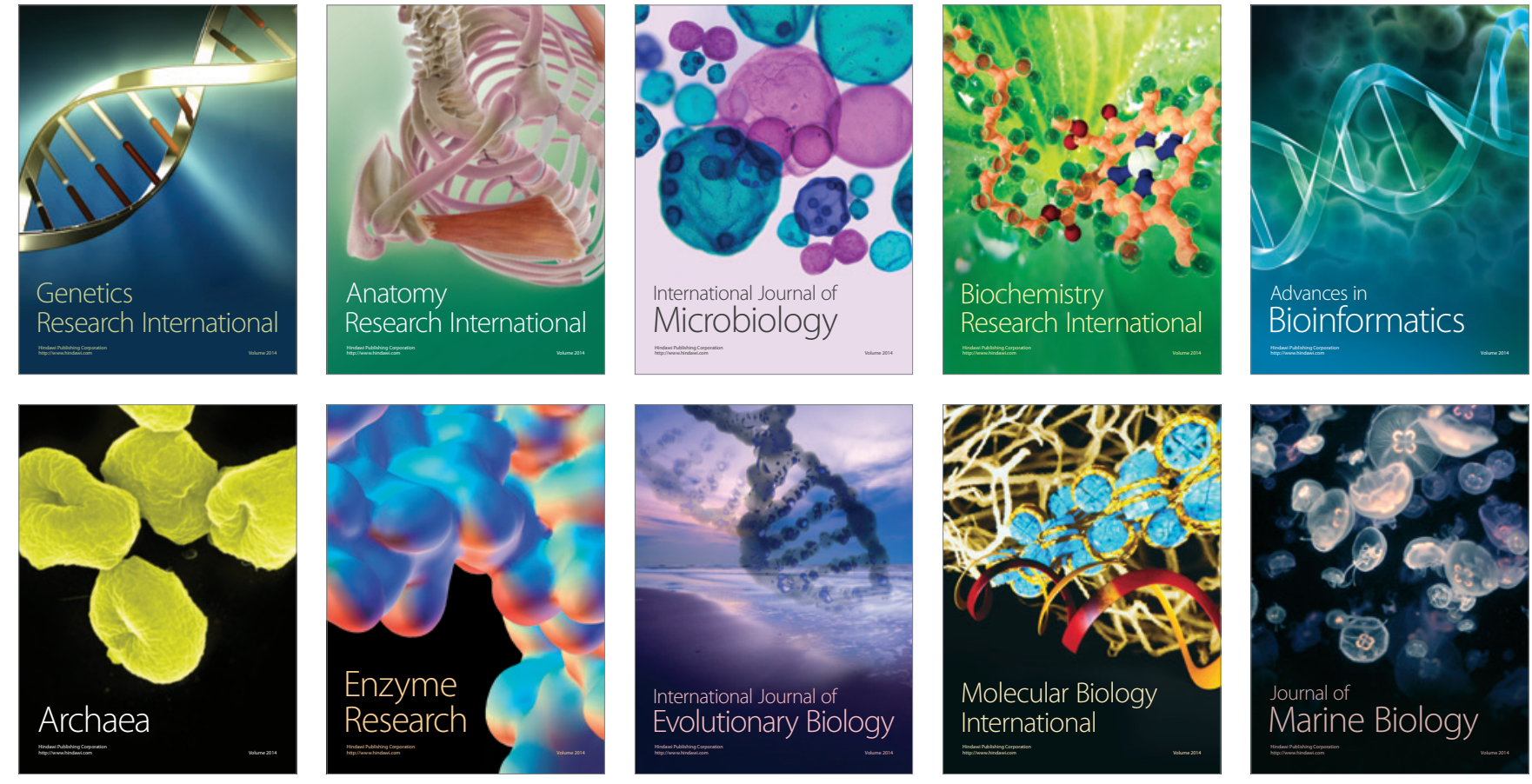